\title{
Human Papillomavirus 16 L1 Protein Expression and Self-Assembly in Recombinant Pichia pastoris
}

\author{
Jing Zhao', Ze-jian Wang ${ }^{1 *}$, Hai-feng Hang ${ }^{1}$, Mei-jin Guo ${ }^{1 *}$, Ying-ping Zhuang ${ }^{1}$, Ju Chu ${ }^{1}$, Si-liang Zhang ${ }^{1}$ and Jue-ren Lou ${ }^{2}$
}

${ }^{1}$ State Key Laboratory of Bioreactor Engineering, East China University of Science and Technology, Shanghai 200237, P.R. China

${ }^{2}$ No. 2 Research Laboratory, Shanghai Institute of Biological Products Co., Ltd., Shanghai 200052, China

\begin{abstract}
Human papillomavirus virus-like particles (HPV VLPS) have been shown to be effective in preventing cervical cancer when used as a prophylactic vaccine. However, little attention has been paid to the process of L1 expression and self-assembly of VLPs in vivo. In this study, the methanol concentration is optimized during fermentation to optimize L1 expression and self-assembly into VLPs. HPV 16 L1 was expressed in Pichia pastoris GS115, and successfully self-assembled into HPV 16 VLPs. The results demonstrate that there is a strong correlation between methanol concentration and the expression of HPV L1 as well as VLP formation. During the methanol induction phase, low concentrations of residual methanol $(0.1-0.3 \%)$ promoted the expression of free L1 and increased the total content of $L 1$, whereas a higher residual methanol concentration $(0.32-0.6 \%)$ was conducive for the self-assembly of LI into VLPs. To conclude, if the residual methanol concentration is too low or too high, the levels of L1 can easily decrease during the fermentation process.
\end{abstract}

Keywords: Recombinant Pichia Pastoris; Human Papillomavirus Virus-like particles; Methanol concentration optimization; Monomer type 16; Self-assembly

\section{Introduction}

Cervical cancer has become the second largest cause of cancer death among women after breast cancer [1]. Human papillomavirus (HPV) is the main etiological agent of cervical cancer, as persistent infection with oncogenic HPV is necessary for the development of cervical cancer. In recent years, several large clinical trials have shown that HPV viruslike particles (VLPs) can been prepared into prophylactic vaccines and provide a sustained immune response against HPV infection. It is estimated that the development of this safe and efficient vaccine can reduce the worldwide incidence of cervical cancer by $70 \%$ through the universal immunization of preadolescent girls [2].

The HPV capsid protein consists of the major capsid protein L1 (comprises $80 \%$ of the capsid protein) and minor capsid protein L2 $[3,4]$. L1 monomers can be expressed in eukaryotic systems such as insect and yeast cells and can self-assemble into virus-like particles $[5,6]$. Due to their strong hydrophobicity, L1 monomers rely on interactions between an $\alpha$-helix, $\beta$-fold, and a $\beta$-loop forming a hydrophobic region between the monomers, which combine to form pentamers. Seventytwo pentamers then combine by disulfide bonds to form a complete, highly symmetrical icosahedron, referred to as an HPV virus-like particle (HPV VLP) $[7,8]$. The assembly of VLPs consisting of HPV L1 is a reversible process. For example, changes in $\mathrm{pH}$, ionic strength, and/ or redox potential can result in the depolymerization and reassembly of VLPs [9]. These self-assembling VLPs can mimic the natural virus to a certain extent, which is a critical step in the vaccine development process. The morphology and immunogenicity of the self-assembling VLPs is similar to HPV virions; however, the VLPs lack viral DNA, and therefore do not stimulate potentially harmful oncogenes so as to provide a safe immune response. After injection of the vaccine, the systematic generation of high levels of anti-HPV L1 IgG antibodies can be achieved, providing HPV subtype-specific immunity as well as immunity against related subtypes.

Numerous studies on HPV vaccines have been carried out recent years, with the L1 protein expressed in several expression systems including Escherichia coli, Saccharomyces cerevisiae, insect cellbaculovirus system, plant, and Pichia pastoris [10-14]. However, due to low yields and relatively high cost, the application of the vaccine in developing countries with high HPV infection rates is limited [15]. It is therefore highly desirable to reduce costs and improve vaccine production. Methanol nutritional type Pichia is used in this study because of the powerful genetic techniques available, high expression levels, rapid growth rate on relatively simple media and well-established fermentation technology, coupled with its economy of use [16]. $P$. pastoris can use methanol as its sole carbon source, and can also use methanol to induce the expression of foreign proteins. Therefore, the methanol concentration is one of the key factors that affect protein expression.

Until now, optimized conditions for the expression of HPV VLPs in the yeast $P$. pastoris system have rarely been reported. However, the methanol concentration, $\mathrm{pH}$, and initial inoculum size were shown to affect cell growth and the expression of HPV 16 L1 in Hansenula polymorpha culture [17]. Carbon source components and harvest time were found to be key factors which influence the production of HPV $16 \mathrm{L1}$ in S. cerevisiae culture [18]. Moreover, the codon optimized MO-HPV58 L1 gene enhanced the expression of soluble L1 protein in $S$, cerevisiae [19]. Studies on the self-assembly process of L1 were

*Corresponding authors: Ze-Jian Wan, State Key Laboratory of Bioreactor Engineering, East China University of Science \& Technology P.O.box 329, 130 Meilong Road, Shanghai 20023, People's Republic of China, Fax: +86-21-64252252; E-mail: wangzejian@ecust.edu.cn

Meijin Guo, State Key Laboratory of Bioreactor Engineering, East China University of Science and Technology P.O.box 329, 130 Meilong Road, Shanghai 200237, People's Republic of China, Fax: +86 21 64253702; E-mail: guo_mj@ecust.edu.cn

Received November 30, 2014; Accepted December 02, 2014; Published December 12, 2014

Citation: Zhao J, Wang ZJ, Hang HF, Guo MJ, Zhuang YP, et al. (2014) Human Papillomavirus 16 L1 Protein Expression and Self-Assembly in Recombinan Pichia pastoris. Global J Technol Optim 6: 168. doi: 10.4172/2229-8711.1000168

Copyright: ( 2014 Zhao J, et al. This is an open-access article distributed under the terms of the Creative Commons Attribution License, which permits unrestricted use, distribution, and reproduction in any medium, provided the original author and source are credited. 
conducted in vitro [20,21], and there are currently no reports of in vivo studies of the L1 self-assembly process. In this study, we investigated the effects of the methanol concentration in the fermentation process on the expression of HPV 16 L1 and L1 self-assembly into HPV 16 VLPs in P. pastoris cells in vivo.

\section{Materials and Methods}

\section{Strain}

Recombinant Pichia pastoris GS115/ HPV16L1 with the mut phenotype was genetically constructed based on the Pichia Expression Kit for the expression of recombinant proteins in Pichia pastoris (Invitrogen, CA, USA). Briefly, the synthetic $16 \mathrm{~L} 1$ gene was inserted into pPIC3.5K at the EcoRI and NotI sites to generate pPIC3.5K-HPV16L1. It was then linearized using BglII and electropolated into competent GS115 cells followed by patching onto MD/MM plates. MD medium consisted of 13.4\% Yeast Nitrogen Base without Amino Acids (YNB), $4 \times 10^{-5} \%$ biotin and $2 \%$ glucose, and $\mathrm{MM}$ consisted of $13.4 \% \mathrm{YNB}$, $4 \times 10^{-5} \%$ biotin and $0.5 \%$ methanol. Colonies that grew normally on the MD plate but slowly on the MM plate were of the GS115/pPIC3.5KHPV16L1 strain with the required His ${ }^{+} \mathrm{Mut}^{\mathrm{s}}$ phenotype.

\section{Media}

YPD media was used as plate and seed media, while the fermentation medium was FM21 basal salt containing glycerol $4 \%$ (w/v) and $4 \mathrm{~mL} / \mathrm{L}$ PTM1 trace element solution. Feed growth medium was $50 \%$ glycerol (w/v, containing $12 \mathrm{~mL} / \mathrm{L}$ PTM1). The fermentation induction medium was $100 \%$ methanol containing $12 \mathrm{~mL} / \mathrm{L}$ PTM1.

\section{Methods}

Shake flask culture: In order to maintain consistent physiological characteristics of cells in different shaken flasks, the fermentation broth was taken when the $\mathrm{OD}_{600}$ of cells in a $30 \mathrm{~L}$ bioreactor reached approximately 200 , then $50 \mathrm{~mL}$ of broth was inoculated into a 500 $\mathrm{mL}$ shake flask and incubated for $2 \mathrm{~h}$ to deplete the glycerol. Next, the protein expression phase was initiated by the addition of fermentation induction medium at $12 \mathrm{~h}$ intervals.

Fermentation: A single colony was picked from a fresh YPD plate and inoculated into the seed medium, and cultured at $30^{\circ} \mathrm{C}$ with a shaking speed of $220 \mathrm{rpm}$ for 20 to $25 \mathrm{~h}$ until an $\mathrm{OD}_{600}$ between 10 and 15 was achieved. The entire $1.5 \mathrm{~L} / 4 \mathrm{~L}$ was aseptically transferred into a $30 \mathrm{~L} / 100 \mathrm{~L}$ bioreactor containing $15 \mathrm{~L} / 40 \mathrm{~L}$ of the fermentation medium. The $\mathrm{pH}$ was adjusted to approximately 5.0 with ammonia and kept at 5.0 during the cell growth phase. When the glycerol was completely consumed, dissolved oxygen (DO) levels suddenly increased, followed by a glycerol-fed batch phase. In this phase $50 \%$ (v/v) glycerol was fed until an $\mathrm{OD}_{600}$ between 350 and 400 was reached. The induction was performed with a continuous feed containing $100 \%$ methanol. The feeding rate for methanol was controlled according to the concentration of residual methanol in the fermentation bioreactor. DO levels in the bioreactor were maintained at $20 \%$ to $40 \%$ for the entire fermentation by 1.0-2.0 VVM aeration and $500 \mathrm{rpm}$ agitation speed. Pure $\mathrm{O}_{2}$ could be injected if needed. The fermentation period was approximately $72 \mathrm{~h}$ to $96 \mathrm{~h}$.

Cell optical density $\left(\mathrm{OD}_{600}\right)$ analysis: The $\mathrm{OD}_{600}$ was measured after dilution, and deionized water was used as the control for the colorimetric determination $\left(\mathrm{OD}_{600}=\mathrm{OD}\right.$ reading $\times$ dilution $)$.

Glycerol concentration: The glycerol concentration was analyzed using the sodium periodate titration method as previously described [22].
Methanol concentration: The methanol concentration was detected quantitatively by gas chromatography (GC) analysis as described in our previous publication [23]. FC-2002 was used as an auxiliary to detect the flow of methanol (East China University of Science \& Technology, Shanghai, China).

Semi-quantitative analysis of HPV 16 L1: Cells were mechanically lysed using glass beads and the lysate supernatant was analyzed by SDSPAGE [24], while purified HPV16 L1 with standard concentrations and lysate supernatant samples prior to induction were used as controls. After electrophoresis, the proteins were transferred to a nitrocellulose membrane. A HPV16 L1 monoclonal antibody (purified mouse anti-L1 protein of human papilloma virus; BD Biosciences, USA) was the primary antibody used to recognize and capture HPV16 L1, and this was specific against HPV16 L1. The secondary antibody was an alkaline phosphatase-conjugated goat anti-mouse IgG $(\mathrm{H}+\mathrm{L})$ (Millipore, USA). The relative concentration of monomeric L1 was detected and calculated using a Bio-RAD Chemi Doc XRS with Image Lab Software (Bio-Rad, USA) after using the ECL chromogenic reagent (RPN2232, GE).

Quantitative determination of HPV 16 VLPs (ELISA):_Cells were mechanically lysed using glass beads and centrifuged, the lysate supernatant was retained as the samples for analysis. Samples were then diluted with $1 \% \mathrm{BSA}$, and $100 \mu \mathrm{L}$ of each sample was added to the microporous plate and coated with a monoclonal antibody that was specific against VLPs (SHSO04, SIBP, China). Lysate supernatant samples before induction were used as the negative control. They were then incubated for $60 \mathrm{~min}$ in plates at $37^{\circ} \mathrm{C}$. The liquid was then discarded, and the wells were washed with $1 \times$ washing liquid three times, before being patted dry.

After washing, $100 \mu \mathrm{L}$ of enzyme binding material working fluid was added to each well, incubated for $45 \mathrm{~min}$ in a membrane-sealed plate at $37^{\circ} \mathrm{C}$. Then all liquid was removed and wells were washed with $1 \times$ washing liquid five times, and patted dry after the completion of the last wash. After the washing steps, $50 \mu \mathrm{L}$ of chromogenic substrate solution $\mathrm{A}$ and $50 \mu \mathrm{L}$ of substrate color liquid B was added to each well, mixed gently and incubated at room temperature in the dark for 5 to $10 \mathrm{~min}$. After this, $50 \mu \mathrm{L}$ of terminate liquid was added to each well and gently mixed. The microplate wavelength was $450 \mathrm{~nm}$, the reference wavelength was $630 \mathrm{~nm}$, and the absorbance value (OD) of each well was measured. The results were fitted with a $\log \mathrm{X}-\log \mathrm{Y}$ function.

\section{Results}

\section{Expression of HPV $16 \mathrm{L1}$ in methanol-induced recombinant P. pastoris}

L1 (55 kDa) was expressed and self-assembled into VLPs during the methanol induction phase. Total L1 content was calculated as a relative content and obtained by semi-quantitative Western blotting (Figure 1). Purified HPV 16L1 was used as the standard concentration as a concentration control. Generally, methanol concentration plays a vital role in the expression of foreign proteins in the methylotrophic yeast $P$. pastoris although it is toxic to cells. As shown in Figure 2, in the early methanol induction period, the amount of total L1 rapidly increased when the residual methanol concentration was less than $0.32 \%$. When the residual methanol concentration increased beyond $0.32 \%$, however, the total L1 concentration dropped sharply. Once the residual methanol concentration was $<0.3 \%$, the production of total L1 increased again during this production phase. This result suggests that the content of L1 changed according to variations in the residual 
Citation: Zhao J, Wang ZJ, Hang HF, Guo MJ, Zhuang YP, et al. (2014) Human Papillomavirus 16 L1 Protein Expression and Self-Assembly in Recombinant Pichia pastoris. Global J Technol Optim 6: 168. doi: 10.4172/2229-8711.1000168

Page 3 of 6
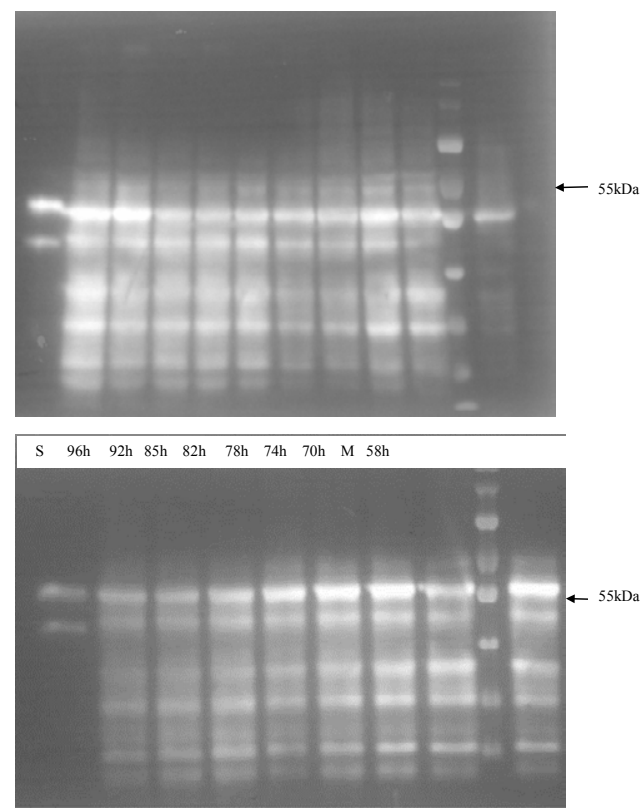

Figure 1:Figure of the Western blot assay to present the expression of HPV 16 L1 in recombinant Pichiapastoris GS115/ HPV16L1 cultured in a $100-\mathrm{L}$ bioreactor. Samples from $34 \mathrm{~h}$ to $96 \mathrm{~h}$ were all lysate supernatants, HPV16 L1 appeared at $55 \mathrm{kDa}$.

$34 \mathrm{~h}$ : Iysate supernatant sample before induction; M: marker (PageRuler ®Prestained Protein Ladder, 10 to $170 \mathrm{kDa}, 26616$, Thermo Scientific Pierce); S: purified HPV16 L1 with standard concentrations.

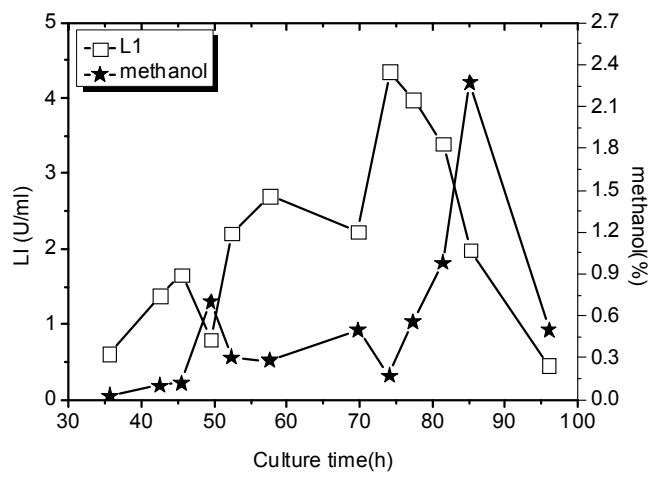

Figure 2:Expression of HPV $16 \mathrm{~L} 1$ and methanol concentration in recombinant Pichiapastoris GS115/ HPV16L1 cultured in a 100-L bioreactor.

Recombinant Pichiapastoris GS115/ HPV16L1 was cultivated in a fedbatch culture. It has two phases: growth phase including a glycerolbatch phase and a glycerol-fed batch phase, and a protein expression phase, which was namely the methanol induction phase that was fed with methanol from $35 \mathrm{~h}$. The feed rate was adjusted based on the residual methanol concentration in the broth. “ $\square$ : HPV16L1 production concentration; $\star$ : residual methanol concentration"

methanol concentration. This demonstrates that the L1 content was closely related to the residual methanol concentration. The threshold value of the residual methanol concentration was approximately $0.32 \%$ to achieve a high-level content of L1.

\section{HPV 16 L1 expression and self-assembly during methanol-} induced recombinant $P$. pastoris fermentation

During the expression time course of HPV $16 \mathrm{~L} 1$ in recombinant $P$. pastoris cultured in a 100-L bioreactor (Figure 3), L1 was expressed and self-assembled into VLPs with increasing methanol concentrations. When the $\mathrm{OD}_{600}$ was less than 360 , and residual methanol concentrations were below $0.32 \%$, the content of total L1 and the assembly of VLPs both increased. If the residual methanol concentration was higher than $0.32 \%(50 \mathrm{~h}, 0.7 \%)$, the $\mathrm{L} 1 \%$ (percentage of $\mathrm{L} 1$ of the total protein) drastically reduced. Additionally, cell growth was inhibited as observed by a reduction of biomass yield and the concentration of VLPs decreased for depolymerization. When the $\mathrm{OD}_{600}$ was greater than 360 , the content of total L1 increased rapidly, provided the residual methanol concentration was maintained below $0.3 \%$. Furthermore, the VLPs depolymerized, which means that the level of free L1 expression increased. Possible reasons for this finding are that low concentrations of methanol might only be sufficient for cell growth and L1 expression, and not for VLP self-assembly. If the residual methanol concentration stayed higher than $0.3 \%$, the total L1 content decreased, VLP assembly increased and the L1\% dropped, which indicated that the level of free L1 expression decreased.

It was interesting to note that the number of VLPs would not increase until total $\mathrm{L} 1$ were reduced to below $2 \mathrm{U} / \mathrm{mL}$. This might reflect that a minimum concentration of free $\mathrm{L} 1$ is required for self-assembly. Residual methanol concentration strictly controlled the expression of L1, and also affected the relationship between L1 and VLPs. Therefore, it was necessary to optimize the residual methanol concentration during $P$. pastoris fermentation to express HPV L1.

\section{Optimization of methanol concentrations for expression of HPV 16 VLPs during recombinant $P$. pastoris fermentation}

Initial methanol concentrations of $0.1 \%, 0.2 \%, 0.3 \%, 0.4 \%, 0.5 \%$, $0.6 \%, 0.8 \%$, and $1.0 \%(\mathrm{v} / \mathrm{v})$ were tested for the expression of HPV 16 L1 in flask culture. As shown in Figure 4, the content of L1 reached a maximum at a methanol concentration of $0.2 \%$, while optimal cell growth conditions we observed at $172\left(\mathrm{OD}_{600}\right)$ with a methanol concentration of $0.3 \%$. Once the initial methanol concentration was higher than $0.4 \%$, the L1 content decreased rapidly due to the degradation (L1\%) or formation of insoluble aggregates. However,
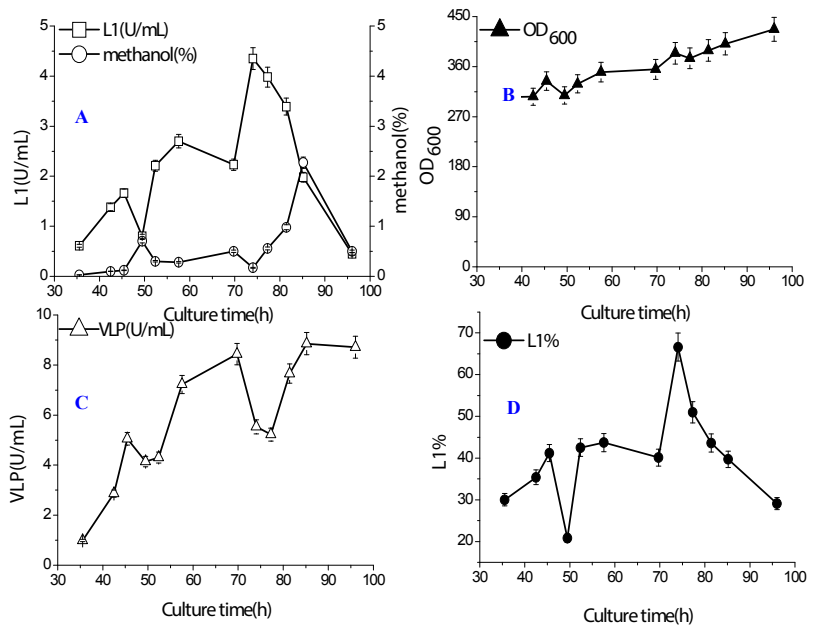

Figure 3: Time-course of HPV $16 \mathrm{~L} 1$ expression by recombinant Figure 3: Time-course of HPV $16 \mathrm{~L} 1$ expression by recombinant $16 \mathrm{~L} 1$ production during methanol induction phase ( $\square$ : HPV16L1 production concentration; o: residual methanol concentration); B: cell growth during the methanol induction phase ( $\boldsymbol{\Lambda}$ : cell optical density determined at the wavelength of $600 \mathrm{~nm}$ ); C: virus-like particle (VLP) level during the methano induction phase $(\Delta:$ VLP concentration); D: HPV $16 \mathrm{~L} 1$ percentage during the methanol induction phase, (๑: HPV 16 L1 percentage). 


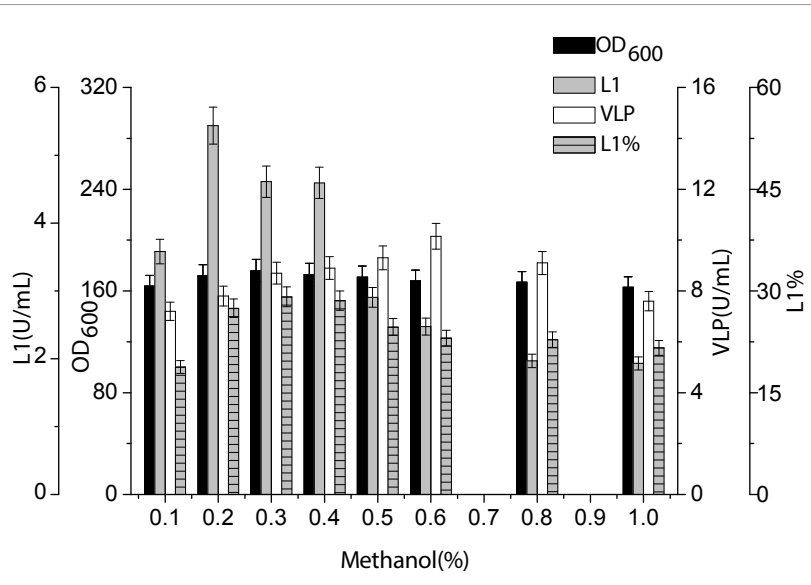

Figure 4: Effect of different methanol concentrations on the expression of HPV 16 L 1 and HPV 16 VLP formation in recombinant Pichiapastoris GS115/ HPV16L1 cells.
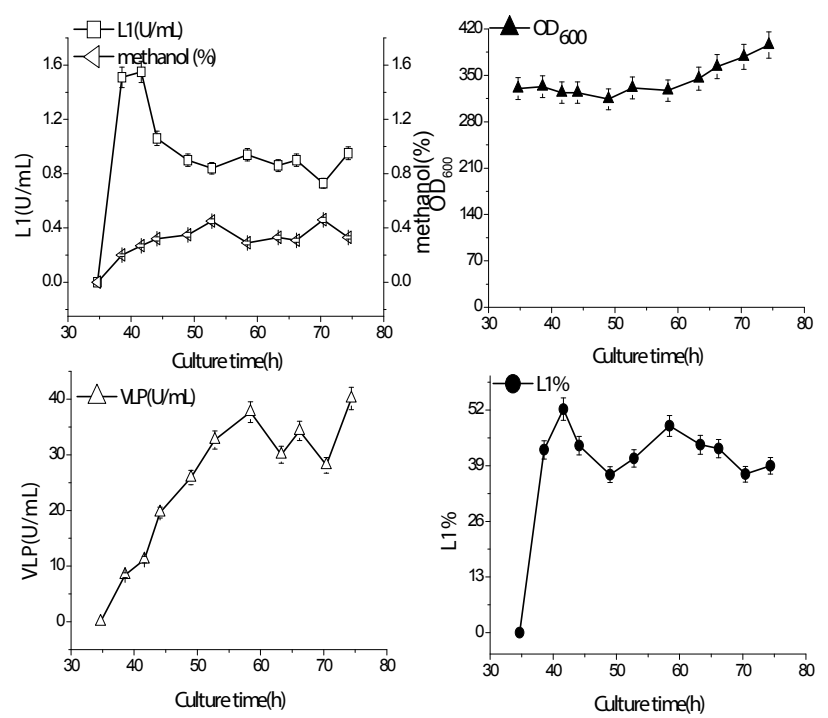

Figure 5: Expression of HPV 16 L1 by recombinant Pichiapastoris GS115/ HPV 16 L1 cultured in a $30-L$ bioreactor during the methanol induction phase. A: HPV 16 L1 production during the methanol induction phase ( $\square$ : HPV $16 \mathrm{~L} 1$ production concentration; $\triangleleft$ : residual methanol concentration); B: cell growth during the methanol induction phase $(\boldsymbol{\Delta}$ : cell optical density determined at the wavelength of $600 \mathrm{~nm}$ ); C: virus-like particle (VLP) level during the methanol induction phase ( $\triangle$ : VLP concentration); D: HPV 16 L1 percentage during the methanol induction phase, ( $\bullet$ HPV16L1 percentage).

VLP assembly showed different optimal conditions, as higher residual methanol concentrations were more conducive to VLP assembly. The initial concentration of $0.6 \%$ appeared to be optimal, while the residual methanol was measured at $0.35 \%$ (Table 1 ). When the initial methanol concentration was higher than $0.8 \%$ while the residual methanol concentration was $0.61 \%$, the content of VLPs decreased. This finding strongly suggests that the residual methanol concentration is closely related to the expression of HPV L1 and the assembly of HPV VLPs. In summary, residual methanol concentrations that range from $0.1 \%$ to $0.3 \%$ optimally increase the total L1 content, while concentrations of $0.35 \%$ to $0.6 \%$ improve VLP self-assembly.

\section{Expression of HPV $16 \mathrm{~L} 1$ in recombinant $P$. pastoris cultured in a $30 \mathrm{~L}$ bioreactor}

To verify the influence of residual methanol concentrations on L1

\begin{tabular}{|c|c|c|c|c|}
\hline $\begin{array}{c}\text { Initial methanol } \\
\mathbf{( \% )}\end{array}$ & $\begin{array}{c}\text { Residual } \\
\text { methanol (\%) }\end{array}$ & $\begin{array}{c}\text { Content of total } \\
\mathbf{L 1}(\mathbf{U} / \mathbf{m l})\end{array}$ & $\begin{array}{c}\text { Concentration of } \\
\text { VLPs(U/ml) }\end{array}$ & L1\% \\
\hline 0.1 & 0 & 3.61 & 7.22 & 17.06 \\
\hline 0.2 & 0 & 5.35 & 7.81 & 27.38 \\
\hline 0.3 & 0.08 & 4.59 & 8.73 & 29.87 \\
\hline 0.4 & 0.19 & 4.53 & 8.91 & 28.96 \\
\hline 0.5 & 0.3 & 2.92 & 9.29 & 24.31 \\
\hline 0.6 & 0.35 & 2.37 & 10.15 & 22.85 \\
\hline 0.8 & 0.61 & 1.96 & 9.12 & 22.71 \\
\hline 1.0 & 0.86 & 1.91 & 7.63 & 20.52 \\
\hline
\end{tabular}

Table 1: Influence of methanol concentration on recombinant Pichia pastoris expression of HPV 16 L1.

expression and self-assembly into VLPs, fermentation was carried out in a $30 \mathrm{~L}$ bioreactor. The strategy was to maintain residual methanol concentrations below $0.3 \%$ in the early induction period to enhance the content of L1, while later increasing the residual methanol concentration to between $0.3 \%$ and $0.5 \%$ to promote $\mathrm{L} 1$ self-assembly into VLPs. Similarly, residual methanol concentration affected L1 expression and self-assembly (Figure $5 \mathrm{~A}$ and $5 \mathrm{~B}$ ). When the residual methanol concentration was kept below $0.3 \%$, L1 was expressed quickly and the total $\mathrm{L} 1$ reached a maximum while simultaneously selfassembling into VLPs. When the residual methanol concentration was kept between $0.32 \%$ and $0.45 \%, \mathrm{~L} 1$ content decreased sharply and the L1\% dropped rapidly. Furthermore, the content of VLPs rose, which confirmed that the expression of free L1 decreased. As the observed rate of VLP concentration showed a distinct linear upward trend, the L1 assembly speed was so rapid that some insoluble aggregates might have formed due to incomplete or improper folding, so as to reduce the total concentration of L1. After that, cells began to grow rapidly and the VLP content remained stable. It is possible that, at this point, there was no further expression of free L1.

This experiment provides strong evidence that lower concentrations of residual methanol $(0.1-0.3 \%)$ promoted L1 expression and a higher concentration of residual methanol $(0.32-0.6 \%)$ effectively promoted L1 monomer self-assembly into VLPs. The L1 content can be sensitive to the residual methanol concentration. As such, the feed strategy according to the optimized residual methanol concentrations can be applied to improve the expression of L1 and VLP production.

\section{Discussion}

In this study, we investigated the effects of residual methanol concentration on the expression of HPV L1 during fermentation and self-assembly of L1 monomers into VLPs using recombinant $P$. pastoris. When $P$. pastoris is used for the expression of exogenous protein, methanol acts both as a carbon and energy source for cell growth and metabolism, as well as an induction signal for recombinant gene expression. Suboptimal methanol concentrations in the fermentation broth are not conducive to the expression of the exogenous protein. Effective control of the residual methanol concentration in the process of induction culture will significantly affect the efficient expression of HPV L1 in the P. pastoris expression system.

We induced cultures in both $30 \mathrm{~L}$ and $100 \mathrm{~L}$ bioreactors, and discovered a strong correlation between L1 expression, VLP selfassembly and residual methanol concentration (Figure 1, Figure 4A), and that $\mathrm{L} 1$ expression was sensitive to changes in residual methanol concentration. Low residual methanol concentrations can promote the expression of L1. Once the residual methanol concentration was greater than $0.32 \%$, the L1 content dropped sharply. Some studies suggest that proteases and lack of a nitrogen source are the primary 
Citation: Zhao J, Wang ZJ, Hang HF, Guo MJ, Zhuang YP, et al. (2014) Human Papillomavirus 16 L1 Protein Expression and Self-Assembly in Recombinant Pichia pastoris. Global J Technol Optim 6: 168. doi: 10.4172/2229-8711.1000168

causes of recombinant protein degradation $[25,26]$. On the other hand, L1 monomers formed some larger insoluble polymers and aggregates during the self-assembly process, which were removed by centrifugation after cell disruption [18], and resulted in a decrease in total L1 content. When residual methanol concentration was maintained above $0.32 \%$, although the L1 content was reduced, the yield of HPV VLPs increased. At high residual methanol concentrations, L1 monomers were more conducive to the assembly of VLPs. Possible reasons were that higher residual methanol concentrations in the culture could meet the energy demand for $\alpha$-helix, $\beta$-folding and disulfide-bond formation in the L1 self-assembly process. The optimization of methanol concentration experiments in shake flasks and the verification in $30 \mathrm{~L}$ bioreactor culture further confirmed that low residual methanol concentrations are conducive to $\mathrm{L} 1$ expression and a higher concentration of residual methanol will promote L1 self-assembly into VLPs (Figure 3). Also, the feed strategy along with optimized methanol concentrations can effectively improve the production of L1 and VLPs.

When the initial methanol concentration was too low $(<0.2 \%)$, methanol was quickly consumed and became the rate-limiting substrate, so that the carbon source and energy requirements for cell growth and L1 expression could not be fulfilled. The methanol was used by $P$. pastoris primarily for cell growth and not for the expression of L1. Simultaneously, L1 degraded more rapidly and the total content was lower. When too much methanol was added, residual concentrations of methanol increased accordingly. However, high residual methanol concentrations $(>0.32 \%)$ could inhibit the activity of some key enzymes in the process of methanol metabolism, such as alcohol oxidase, which not only restrained cell growth but also reduced the expression of L1 [27].

Intracellular free L1 would no longer self-assemble into VLPs below a threshold concentration during the fermentation process. Ding et al. simulated the assembly and aggregation of VLPs in vitro [28], and found that there was a critical concentration required for the assembly (also called the minimum assembly concentration) of capsomeres. When the capsomere concentration dropped below a certain value, VLP assembly ceased. Mukherjee et al. (HPV 16 VLPs), and Casini et al. [29] (HPV 11 VLPs) also mentioned this critical assembly concentration in their studies on VLP assembly in vitro. Therefore, improving the concentration of free L1 is an encouraging strategy to increase the yield of HPV VLPs.

\section{Conclusion}

In this study, we have determined an optimal fermentation strategy for production of HPV VLPs. During the early induction period, lower residual methanol concentrations (0.1-0.3\%) are used to rapidly express and accumulate $\mathrm{L} 1$, and increase the cell biomass. Then, increasing residual methanol concentrations to between $0.32 \%$ and $0.6 \%$ promotes L1 self-assembly into HPV VLPs. This research lays an important foundation for fermentation optimization for the production of HPV VLPs and provides a greater understanding of the expression of intracellular L1 as well as the characteristics of L1 selfassembly into VLPs. Improving the yield of HPV VLPs is a significant step toward reducing the cost of the HPV vaccine, and will benefit developing countries with high HPV infection rates.

\section{Authors' Contributions}

Jing Zhao carried out the fermentation and data statistic; Zejian Wang participated in its experimental design, Haifeng Hang participated in on-line fermentation data processing and analysis,
Meijin Guo participated in its design and drafted the manuscript, Yingping Zhuang participated in its design and coordination, Ju Chu critically revised the manuscript, Siliang Zhang participated in analysis and interpretation of data, and Jueren Lou carried out strain construction and final manuscript. All authors read and approved the final manuscript.

\section{Acknowledgement}

This work was financially supported by a grant from the National High Technology Research and Development Program of China (863 Program) (2012AA02A401, 2012AA021201, and 2014AA021705).

\section{References}

1. Saha A, Chaudhury AN, Bhowmik P, Chatterjee R (2010) Awareness of cervical cancer among Female students of premier colleges in Kolkata. India Asian Pac. J Cancer P 11: 1084-1090.

2. Frazer IH (2008) HPV vaccines and the prevention of cervical cancer. Update On Cancer Therapeutics 3: 43-48.

3. Hernandez J, Elahi A, Siegel E, Coppola D, Riggs B, et al. (2011) HPV L1 capsid protein detection and progression of anal squamous Neoplasia, Am J Clin Phol 135: 436-441.

4. Ma B, Roden RB, Hung CF, Wu TC (2011) HPV pseudovirions as DNA delivery vehicles, Ther. Deliv 2: 427-430.

5. Zhao Q, Modis Y, High K, Towne V, Meng Y, et al. (2012) Disassembly and reassembly of human papillomavirus virus-like particles produces more virionlike antibody reactivity, Virol J 9: 52

6. Pandhi D, Sonthalia S (2011) Human papilloma virus vaccines: Current scenario, Indian J Sex Transm Dis 32: 75-85.

7. Modis $\mathrm{Y}$, Trus BL, Harrison SC (2002) Atomic model of the papillomavirus capsid, EMBO J 21: 4754-4762.

8. Chen XS, Garcea RL, Goldberg I, Casini G, Harrison SC (2000) Structure of small virus-like particles assembled from the $L 1$ protein of human papillomavirus 16. Mol Cell 5: 557-567.

9. McCarthy MP, White WI, Palmer-Hill F, Koenig S, Suzich JA (1998) Quantitative disassembly and reassembly of human papillomavirus type 11 virus like particles in vitro. J Virol 72: 32-41.

10. Giorgi C, Franconi R, Rybicki EP (2010) Human papillomavirus vaccines in plants, Expert Rev. Vaccines 9: 913-924.

11. Schiller JT, Okun MM (1996) Human papillomavirus vaccines: current status and future prospects, Drugs 70:1079-1098.

12. Berg M, Gambhira R, Siracusa M, Hoiczyk E, Roden R (2007) HPVI6 L1 capsid prorein expressed from viable adenovirus recombinants elicits neutralizing antibody in mice, Vaccine 25: 3501-3510.

13. Aires KA, Cianciarullo AM, Carneiro SM, Villa LL, Boccardo E, et al. (2006) Production of human papillomavirus type $16 \mathrm{~L} 1$ virus-like particles by recombinant Lactobacillus casei cells, Appl Environ Microbiol 72: 745-752.

14. Senger T, Schädlich L, Gissmann L, Müller M (2009) Enhanced papillomaviruslike particle production in insect cells, Virology 388: 344-353.

15. Schadlich L, Senger T, Kirschning CJ, Muller M, Gissmann L (2009) Refining HPV 16 L1 purification from E. coli: reducing endotoxin contaminations and their impact on immunogenicity, Vaccine 27: 1511-1522.

16. Bazan SB, Chaves AAM, Aires KA, Cianciarull AM, Garcea RL, et al. (2009) Expression and characterization of HPV-16 L1 capsid protein in Pichia pastoris, Arch. Virol 154: 1609-1617.

17. Li W, He X, Guo X, Zhang Z, Zhang B (2009) Optimized expression of the L1 protein of human papillomavirus in Hansenula polymorpha, Chin. J Biotech 25 : 1516-1523.

18. Kim HJ, Kwag HL, Jin YJ, Kim HJ (2011) The composition of the carbon source and the time of cell harvest are critical determinants of the final yield of human papillomavirus type $16 \mathrm{~L} 1$ protein produced in Saccharomyces cerevisiae. Protein Expr. Purif 80: 52-60.

19. Kim HJ, Kwag HL, Kim HJ (2013) Codon optimization of the human papillomavirus type $58 \mathrm{~L} 1$ gene enhances the expression of soluble L1 protein 
Citation: Zhao J, Wang ZJ, Hang HF, Guo MJ, Zhuang YP, et al. (2014) Human Papillomavirus 16 L1 Protein Expression and Self-Assembly in Recombinant Pichia pastoris. Global J Technol Optim 6: 168. doi: 10.4172/2229-8711.1000168

Page 6 of 6

in Saccharomyces cerevisiae Biotechnol. Lett 35: 413-421.

20. Mukherjee S, Thorsteinsson MV, Johnston LB, DePhillips PA, Zlotnick A (2008) A quantitative description of in vitro assembly of human papillomavirus 16 virus-like particles. J Mol Biol 381: 229-237.

21. Xie L, Smith GR, Feng X, Schwartz R(2012) Surveying capsid assembly pathways through simulation-based data fitting, Biophys J 103: 1542-1554

22. Kang H, Liu GJ, Sun FQ, Zhao X (2010) Determination of glycerol conten in clavulanic acid fermentation broth by titration, He bei Journal of Industrial Science and Technology 27: 189-191.

23. Deng BB, Fang HQ, Xue C, Zhao HL, Liu ZM (2001) GC rapid analysis of methanol and ethanol during high cell density culture of recombinant methylotrophic yeast. Chin. Ind. Microbiol 31: 26-29.

24. Liu D, Cai J, Xie CC, Liu C, Chen YH (2010) Purification and partial characterization of a $36-\mathrm{kDa}$ chitinase from Bacillus thuringiensis subsp, colmeri, and its biocontrol potential. Enzyme Microb. Technol 46: 252-256.
25. Korayashi K, Kuwae S, Ohya T, Ohda T, Ohyama M, et al. (2000) High level expression of recombinant human serum albumin from the methylotrophic yeast pichia pastoris with minimal protease production and activation, $\mathrm{J} \mathrm{Biosci}$ Bioeng 89: 45-61.

26. Potvin G, Zhang ZS (2010) Strategies for high-level recombinant protein expression in transgenic microalgae: A review. Biotechnol. Adv 28: 910-918.

27. Zutphen TV, Baerends R, Susanna K, Jong A, Kuipers OP, et al. (2010) Adaptation of Hansenula polymorpha to methanol: a transcriptome analysis. BMC Genomics 11: 1-12

28. Ding Y, Chuan YP, He LZ, Middelberg APJ (2010) Modeling the competition between aggregation and self-assembly during virus-like particle processing, Biotechnol. Bioeng 107: 550-560.

29. Casini GL, Graham D, Heine D, Garce RL, Wu DT (2004) In vitro papillomavirus capsid assembly analyzed by light scattering, Virology 325: 320-327. 